Canadian

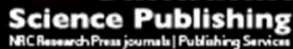

Canadian Geotechnical Journal Revue canadienne de géotechnique

\title{
Experimental and analytical study of seismic site response of discontinuous permafrost
}

\begin{tabular}{|r|l|}
\hline Journal: & Canadian Geotechnical Journal \\
\hline Manuscript ID & cgj-2015-0223.R2 \\
\hline Danuscript Type: & Article \\
\hline Complete List of Authors: & $\begin{array}{l}\text { Dadfar, Behrang; Western University, Department of Civil and } \\
\text { Environmental Engineering } \\
\text { El Naggar, M. Hesham; Western University, Department of Civil and } \\
\text { Environmental Engineering } \\
\text { Nastev, Miroslav; Natural Resources Canada, Geological Survey of Canada }\end{array}$ \\
\hline Keyword: & $\begin{array}{l}\text { site response analysis, cold regions, discontinuous permafrost, shaking } \\
\text { table, numerical modelling }\end{array}$ \\
\hline & \\
\hline
\end{tabular}

SCHOLARONE ${ }^{\text {m }}$

Manuscripts 


\section{Experimental and Analytical Study of Seismic Site Response of Discontinuous Permafrost}

\section{1- Behrang Dadfar}

PhD candidate, Department of Civil and Environmental Engineering, Western University, London, ON, Canada N6A 5B9, sdadfar@uwo.ca

\section{2- M. Hesham El Naggar}

Professor, Department of Civil and Environmental Engineering, Western University, London, ON, Canada N6A 5B9, helnaggar@eng.uwo.ca

\section{3- Miroslav Nastev}

Research scientist, Geological Survey of Canada, Natural Resources Canada, 490, Rue de la Couronne, Québec, QC, Canada G1K 9A9, miroslav.nastev@rncan-nrcan.gc.ca

\section{Corresponding author :}

Name: Behrang Dadfar

Address: Department of Civil and Environmental Engineering, Western University, London, ON, Canada N6A 5B9

Telephone number: 519-701-5916

Email: sdadfar@uwo.ca 


\begin{abstract}
Seismic site response of discontinuous permafrost is discussed. The presence of frozen ground in soil deposits can significantly affect their dynamic response due to stiffer conditions characterized by higher shear wave velocities compared to unfrozen soils. Both experimental and numerical investigations were conducted to examine the problem. The experimental program included a series of $1 \mathrm{~g}$ shaking table tests on small-scale models. Nonlinear numerical analyses were performed employing the FLAC software. The numerical model was verified using the obtained experimental results. Parametric simulations were then conducted using the verified model in order to study the variations of the free-field spectral accelerations (on top of the frozen and unfrozen soil blocks) with the scheme of frozen/unfrozen soil, and to determine the key parameters and their effects on the seismic site response. The results showed that spectral accelerations were generally higher in frozen soils than in unfrozen soils. It was found that the shear wave velocity of the frozen soil, the assumed geometry of the blocks and their spacing have significant impact on the site response.
\end{abstract}

Key words: site response analysis, cold regions, discontinuous permafrost, shaking table, numerical modelling 


\section{Introduction}

Surface seismic ground motions are significantly influenced by local site conditions, e.g., surficial soil or bedrock conditions, depth and geometry of the sedimentary basin, topography, and by the characteristics of the incoming seismic waves. These parameters modify the amplitude, frequency content and duration of the bedrock motion in such a way that bedrock and surface motions are incoherent. The impact of these parameters on the seismic site response is referred to as local site effects. In discontinuous permafrost regions, the site effects can be accentuated by the intermittent presence of frozen soils. Permafrost or perennially frozen ground is a term used to describe the thermal condition of soils when their temperature remains continuously below $0^{\circ} \mathrm{C}$ for a number of years (Muller 2008). In the discontinuous zone, some portions of the soil mass are under frozen conditions whereas others are not. Discontinuous permafrost represents a particular challenge for geotechnical earthquake engineering since frozen soils have different geotechnical properties and relatively higher shear wave velocities than unfrozen soils. The relatively high impedance contrast between the frozen and unfrozen soils in the lateral and/or vertical directions may contribute to important site effects.

Only a limited number of studies considering the effects of permafrost on the free-field ground motion are found in the literature. These are mainly recent studies conducted in response to infrastructure developments in cold regions, mostly transportation systems and energy pipelines. Among the first studies is the investigation conducted by Finn and Yong (1978) and Finn et al. (1978), which focused on the seismic behaviour of frozen soils and liquefaction mechanisms in thawed layers. The authors concluded that the simultaneous presence of frozen and unfrozen soils increases the complexity of the free-field ground motion, in particular saturated unfrozen cohesionless soils sandwiched between a frozen surficial layer and underlying permafrost could potentially cause ground instabilities during earthquakes. Characterisation of ground motions at permafrost sites along the Qinghai-Tibet railway, China, was carried out by Wang et al. (2009). They 
conducted numerical simulations employing synthetic input seismic motions with different exceedance probabilities to investigate the influence of ground temperature on the free-field ground motion parameters (acceleration, velocity, displacement and predominant period). Yang et al. (2011) performed one dimensional equivalent linear analysis of vertically propagating horizontal shear waves in order to investigate the effects of permafrost on the seismic response of bridges in Alaska. The effects of variations in permafrost thickness and depth, and depth to bedrock were studied. They concluded that the presence of continuous permafrost remarkably changes the ground motion and should be considered in the seismic design of structures.

All cited site response studies investigated the dynamic behaviour of frozen soils under continuous permafrost conditions. To date, however, there is no numerical or experimental published research, focusing on the soil dynamic behaviour under discontinuous permafrost conditions in the literature.

The objective of this paper is to fill in the current knowledge gap related to the seismic site response under complex discontinuous permafrost conditions by conducting experimental and numerical analyses. Particular attention was given to the dynamic interaction between the portions of frozen soil and the surrounding unfrozen soil, as shown in Fig. 1. Nonlinear models were developed and validated against laboratory tests and sensitivity analysis conducted. The calibrated models were then used to run parametric studies in an effort to quantify the interaction. The findings of this study are important for the safety of infrastructure in discontinuous permafrost regions.

Fig. 1: Dynamic interaction between frozen soil blocks (in gray color) and the surrounding unfrozen soil.

\section{Physical modelling with reduced scale $1 \mathrm{~g}$ shaking table tests}

The scaling relations map geometry, kinematics and dynamics of prototypes to those of models. They can be established by dimensional analysis, similitude theory and the method of governing 
equations (Kline 1965). As it is usually not feasible to fulfill all the similitude requirements, it is preferred to satisfy as many relations as possible giving priority to those relevant to the desired aspects of the problem. Based on the level of similarities, the model can be referred to as "true", "adequate" or "distorted" where, the true model has the highest level of similarity and the distorted model has the lowest (Moncarz and Krawinkler 1981).

Shaking table tests in $1 \mathrm{~g}$ gravitational field are useful tools for studying the behaviour of soils and structures under seismic loading. Full-scale models on a shaking table can "truly" simulate the prototype response. Small-scale models, on the other hand, depending on the degree of satisfaction of the scaling relations, can predict the response quantitatively or qualitatively.

The constitutive behaviour of soil affects considerably the ground deformations. In dynamic problems, the undrained constitutive behaviour of cohesionless soils depends on the confining pressure and density. Due to the smaller confining pressures in the model soils, the stress-strain relations of prototype and model may become different when identical soil densities are considered (contractive and dilative behaviours). Verdugo and Ishihara (1996) demonstrated this effect conducting undrained tests on Toyoura sands with different void ratios and confining pressures. Rocha (1957) presented similarities under total and effective stress conditions for problems involving elastic deformations. The author proposed scaling of the soil constitutive model according to the stress and strain scales. Considering Rocha's assumptions, Iai (1988) further derived scaling relations for soil-structure-fluid modelling in the elastic range. To deal with soil nonlinearities such as large deformations during liquefaction, Roscoe (1968) applied concepts of critical soil mechanics and expressed the conditions of similarity based on the state parameters of prototype and model soils. The theoretical developments were with a few experiments. Towhata (2008) used the experimental results of Verdugo and Ishihara (1996) and Vargas-Monge (1998) to extend the similarity relationships within the full range of soil nonlinearity. To this end, the concept of 
brittleness index (Bishop et al. 1971) was suggested in replication of the strain softening of the constitutive model. Based on this approach it is not necessary to satisfy the similarity of the soil density and a looser soil can simulate the stress-strain relation of the prototype. However, to date there are no definitive scaling relations for the density of sand in the scaled models (Alam and Towhata 2008).

The primary goal of this research is to study the soil-permafrost interaction which heavily depends on the stiffness of both media expressed with respective shear wave velocities. It was therefore decided to keep constant the dimensionless ratios of the shear wave velocities of frozen and unfrozen material measured in the field and in the scaled model. The primary simulation condition is satisfied when applying the field soil density in the model. However, introducing loose soils to satisfy the secondary effects of liquefaction will violate the primary similarity. In addition, preparing saturated experimental models consisting of a combination of high density material (representing permafrost) and low density soil (representing unfrozen soil) is technically difficult. Therefore, the scaling relations of Iai (1988) were preferred to produce "adequate" scaled models with primary focus on the soil-permafrost interaction.

\subsection{Scaling relations}

Similitude relations developed by Iai (1988) for reduced scale models of saturated soil-structurefluid interaction tests at $1 \mathrm{~g}$ gravitational field were used in this study. By satisfying the basic equations of the saturated soil-structure-fluid system for both the model and the prototype, Iai obtained the scaling relations. The basic equations were derived assuming that the soil skeleton is a continuum and the soil displacements and the skeleton strains are small. Iai showed that the similitude relations give good approximation for seismic deformations of the prototype soilstructure. The applied similitude relations and corresponding scaling factors are shown in Table 1. As it can be noted from Table 1, only two of the scaling factors are independent, i.e., the length and the density scale factors $\left(\lambda_{\mathrm{L}}\right.$ and $\lambda_{\rho}$ ), whereas the remaining factors are related to one or both of 
them. Considering the capabilities of the available experimental facility and some additional technical considerations explained below, the appropriate scale factors for the length and density were selected to be 100 and 1 , respectively.

\subsection{Shaking table and soil container}

The shaking table tests were conducted on soil models enclosed in a laminar soil container placed on a $1.22 m \times 1.22 m$ 1D shaking table at the University of Western Ontario, Canada. The shaking table can be excited by either electrical or hydraulic actuator controlled by a digital control module, which allows simulation of various types of dynamic displacement time-histories. In this study, an electrical actuator was used because of the high frequency range of the scaled input motion. The electrical actuator has a maximum displacement stroke of $12 \mathrm{~mm}$ and can generate up to $3 \mathrm{kN}$ base shear within the broad frequency range of 1-150Hz. Scaled records of El Centro Earthquake (1940) were used as an input motion at the base of the shaking table. Each model was excited by three levels of shaking intensity: low $(\mathrm{PGA}=0.15 \mathrm{~g})$, medium $(\mathrm{PGA}=0.3 \mathrm{~g})$ and high $(\mathrm{PGA}=0.5 \mathrm{~g})$. The original duration of the record was $30 \mathrm{sec}$, which in accordance with the similitude relation for time was approximately reduced to $1 \sec$ (Table 1).

The infinite boundaries in the physical model and the $1 \mathrm{D}$ vertical shear-wave propagation were simulated by containing the soil models in laminar (flexible) soil container which does not impose unrealistic rigid boundary conditions and reduces the reflection of the dynamic waves back into the box. The laminar container comprised 12 horizontal lamina supported individually on linear bearings and steel guide rods connected to an external frame as shown in Fig. 2. The inner dimensions of the container are $404 \mathrm{~mm}, 900 \mathrm{~mm}$ and $450 \mathrm{~mm}$ corresponding to the height, length and width, respectively. The laminar container does not have a bottom plate, allowing the soil to rest on the shaking table directly. Further details about specifications and fabrication of this container can be found in Turan et al. (2009). The test setup included the shaking table, the flexible container, the electric control module and the data acquisition system as shown in Fig. 2. 
Fig. 2: Test setup including (from left to right): the data acquisition system, the electric control module, the shaking table with the mounted flexible container with 12 frames; and sand-cement blocks representing frozen soils.

\subsection{Material properties and preparation}

Sand: North America permafrost has often formed of cohesionless soils (Finn and Yong 1978). For this study, the soil types and stratigraphy were selected based on the information in the borehole database of the Yukon-Alaska highway and of the pipeline projects along the Mackenzie Valley/Delta region in Canada and the US (Yukon Geological Survey 2014; Geological Survey of Canada 2014). Both project routes pass through regions with predominantly discontinuous permafrost conditions. To simulate the field soil conditions, a simplified soil profile consisting of clean sand (Ottawa sand) underlain by well-graded sand (construction sand) was considered. The respective gradation curves and geotechnical parameters are shown in Fig. 3 and in Table 2, respectively.

Fig. 3: Particle size distribution curves.

The thickness of the active layer in permafrost is few centimetres to a few metres exposed to seasonal and sometimes daily freeze/thaw cycles (Johnston 1981). Thus, these soils experience microstructural changes as shown by Viklander (1998) and Qi et al. (2008). They demonstrated that initially loose and dense soils (silty soil and silty tail) end up with the same constant residual void ratio after a few freezing cycles. In this study, considering the effect of freeze/thaw cycles on the void ratio and its small range in the Ottawa sand, relative density of 50\% was considered for the surficial active layer.

Frozen soil: Since the investigation of the interaction between the frozen and unfrozen soils is the main goal of this research, only mechanical properties of frozen soil, i.e., shear wave velocity, friction angle and cohesion are considered and those parameters related to the thermal behaviour and long-term mechanical response (creep) are not considered in the scope of this work. Having this in mind and the challenges in working in laboratory temperature with frozen and unfrozen soils at the same time, sand-cement mixture (SCM) was used to represent the blocks of frozen soil. 
SCM is a cured mixture of sand, Portland cement and water that has a higher shear wave velocity compared to the ordinary sand (M. H. El Naggar, A. Saeed and O. Drbe, Static and dynamic properties of fillcrete. Geotechnical Research Centre, Western University, 2013). In addition, the application of the SCM allows the control of the required shear wave velocity and to some extent unconfined compressive strength (in order to satisfy similitude relations) by using a proper mix design.

Most of the ultimate shear strength of frozen soil is provided by cohesion where internal friction has only insignificant role (even in sandy soils) (Tsytovich 1975). The ultimate shear strength of frozen soils, (subjected to normal pressures of up to $2 \mathrm{MPa}$ ), under instant loading can be determined from the Mohr-Coulomb equation:

$$
\tau_{u l t}=c_{\theta}+\sigma \tan \phi_{\theta}
$$

where $\tau_{u l t}$ is the ultimate shear strength, $\sigma$ is the normal stress, $c_{\theta}$ is the cohesion and $\phi_{\theta}$ is the internal friction angle. Subscript $\theta$ for cohesion and friction denotes function of temperature. Table 3 gives examples of the variation of cohesion with temperature for a silty sand with moisture content of $23 \%$, as reported by Tsytovich (1975).

On the other hand, the compressive strength of frozen soils depends on negative temperature, moisture content and their composition and structure (Tsytovich 1975). Results of uniaxial compression tests on structurally undisturbed frozen silty sand sampled from permafrost reported in Tsytovich (1975) are presented in Table 4.

Experiments show that frozen soils have elastic modulus tens to hundreds of times larger than that of unfrozen soils. The elastic modulus of frozen soil is a function of the ice content, negative temperature, external pressure and composition of soils. At the same time, the Poisson's ratio for frozen soils increases from typical values for solids in low temperatures to almost 0.5 for temperatures close to $0^{\circ} \mathrm{C}$ (Tsytovich 1975$)$. 
The ultrasonic studies of Nakano and Froula (1973) on artificially frozen samples of Ottawa sand and Hanover silt and investigations of Zimmerman and King (1986) on undisturbed permafrost soils from the Mackenzie River valley, Beaufort Sea and the Canadian Arctic Islands show typical ranges of shear wave velocity of $1200-1900 \mathrm{~m} / \mathrm{sec}$ for silt and $1750-2500 \mathrm{~m} / \mathrm{sec}$ for sand. Furthermore, the seismic cone penetration tests and seismic tomographic imaging performed by LeBlanc et al. (2004) in a silty sand permafrost at Umiujaq, northern Quebec, Canada, revealed a shear wave velocity between 900 and $1750 \mathrm{~m} / \mathrm{sec}$. Based on the above observations, an average value of $1500 \mathrm{~m} / \mathrm{sec}$ was chosen for the shear wave velocity of the frozen soils.

Viscous fluid: It is not possible to simultaneously satisfy the similitude relations for both "dynamic" and "diffusion" times in small-scale saturated geotechnical models without changing soil permeability or pore fluid viscosity (Muir Wood 2004). The former controls the dynamic aspects of loading and related parameters, whereas the latter regulates the phenomenon of excess pore pressure build-up. In this study, the diffusion time scale was adjusted by increasing the viscosity of the pore fluid. To this end, Glycerine-water solution was used instead of water. After the required value of viscosity was determined from Table 1 , the Glycerine was diluted with water (25\% water, 75\% Glycerine) to reach the target viscosity based on the proportions presented by Cheng (2008).

Preparation of soil model: The bottom layer of construction sand with relative density of $80 \%$ and total height of $20 \mathrm{~cm}$ was placed in the laminar container in 5 successive sub-layers of equal height compacted using the moist compaction method. The overlying layer comprised Ottawa sand and was placed using the same method to a total thickness of $18.75 \mathrm{~cm}$ and relative density of $65 \%$. The top layer, representing the active layer, with thickness of $1.25 \mathrm{~cm}$ and relative density of $50 \%$ was also composed of Ottawa sand and was deposited using the water sedimentation method. A sand pluviator consisting of a funnel, a sieve and two sliding rods was designed for this purpose to move over the flexible container and to uniformly distribute the sand over the desired area. The pluviator was first calibrated with the Glycerine-water solution (the pore fluid) and an appropriate sieve size 
was selected such that the target relative density was attained. Following the preparation of each test model, it was left for 24 hours to ensure that excess pore pressure was dissipated completely.

\subsection{Instrumentation}

A number of accelerometers and miniature pressure transducers were installed in each test model. The accelerometers were fixed to the top layer (by rigid glue to SCM blocks) in order to monitor the ground surface accelerations and one was rigidly attached to the table top to monitor the base input acceleration. The miniature pressure transducers were employed to monitor the changes of pore water pressure. The transducers were small and light-weight enough for the least possible interaction with the surrounding soil. To measure the net water pressure a bronze filter was added to the tip of the transducers. The maximum capacity of the transducers was 1 bar and they were calibrated before application. Technical specifications of the instruments are given in Appendix. A schematic view of the test setup is illustrated in Fig. 4. The geometric parameters of the experiments are presented in Table 5.

Fig. 4: Schematic presentation of the typical test setup (a) longitudinal cross section and (b) top view. Frozen soil blocks are indicated in grey color.

\section{Numerical simulations}

The direct nonlinear method is suitable for site response analysis because of its ability to describe the behaviour of soils subjected to cyclic loads in a realistic manner (Kramer 1996). Important practical issues and developments related to this technique were highlighted by Hashash et al. (2010). FLAC software (Itasca Consulting Group, Inc. 2002) has capabilities to simulate many of the advanced features of the nonlinear dynamic method in the site response analysis. It applies an explicit finite difference scheme to solve the full equations of ground motion in continua. Discontinuous permafrost regions in North America often comprise saturated cohesionless and potentially liquefiable soils (Finn and Yong 1978). Therefore, a representative constitutive model has to be employed to simulate pore water pressure changes in the unfrozen soils during the 
application of seismic loads. The constitutive model proposed by Byrne (1991) that relates the increment of volumetric strain to the cyclic shear strain and uses the Mohr-Coulomb plasticity to define the soil behaviour under effective stress, can be used in FLAC.

The results of the site response experiments conducted in the current study explained some of the effects of a number of parameters and provided data to calibrate and verify the numerical models established using FLAC. The verified numerical models were then used to predict site behaviour in cases that were otherwise not feasible to test in the lab.

The finite difference grid of the model had 468 zones distributed in 13 rows and 36 columns. The width of each zone was $2.5 \mathrm{~cm}$ and their height varied between $1.25 \mathrm{~cm}$ and $5 \mathrm{~cm}$ depending on the location and geometry of the blocks. In this section, assumptions made for the numerical modelling are discussed.

\subsection{Soil stiffness degradation and damping}

The soil stiffness and damping are parameters required for seismic site response analysis. The stiffness of sand deposit is represented by the secant shear modulus $\left(\mathrm{G}_{\mathrm{sec}}\right)$, which varies as a function of the sand relative density, overburden pressure, cyclic strain amplitude, and number of loading cycles for sands (Kramer 1996). There is ample research investigating the soil stiffness degradation (Iwasaki et al. 1978; Kokusho 1980; Seed and Idriss 1970). In contrast, only a few studies considered frozen soils (Singh and Donovan 1977). The results of some of the few studies characterizing degradation of frozen soil are shown in Fig. 5, which demonstrates two degradation curves reported by Seed and Idriss (1970) and Singh and Donovan (1977) for average sand and frozen sand, respectively.

Fig. 5: Shear modulus reduction curves for frozen sand at $-1{ }^{\circ} \mathrm{C}$ by Singh and Donovan (1977) and average unfrozen sand by Seed and Idriss (1970). 
Hardin and Drnevich (1972) developed an equation to describe the shear modulus degradation $\left(\mathrm{G}_{\text {sec }} / \mathrm{G}_{\max }\right)$ which was used to establish EPRI (1993) curves representing the shear modulus reduction under different levels of overburden pressure. This equation is given by:

$$
G_{s e c} / G_{\max }=\left[1+\left(\gamma / \gamma_{r e f}\right)\right]^{-1}
$$

where $\gamma$ and $\gamma_{r e f}$ are the shear strain and the reference shear strain, respectively. In the current study, eq. (2) was employed to model the variation of $\mathrm{G}_{\mathrm{sec}}$ with shear strain. The reference strain, $\gamma_{r e f}$, was varied until the best fit to the target curves shown in Fig. 5 was obtained.

Soil deposits undergoing cyclic loading dissipate energy, which is manifested be their hysteresis loops. The energy dissipation during nonlinear response, defined as material damping, is obtained by computing the area confined by the hysteresis loops. For moderate to high strain levels, the material damping represents the nonlinearity of the material, whereas for low strain levels the damping is assumed to be zero since the material remains in the linear elastic range. However, lab experiments show that even for small strains, soil dissipates some energy and has some form of damping (Zhang et al. 2005). Thus, a minimum value of damping should be usually considered in the analysis. Hence, a $0.5 \%$ Rayleigh damping over the range of predominant frequencies was used in the models (Itasca Consulting Group, Inc. 2002).

\subsection{Excess pore water pressure model}

The changes of pore water pressure can be calculated by employing either the Martin et al. (1975) equation, or the simplified Byrne's formula (Byrne 1991). In the current study, excess pore water pressure build-up under seismic excitation was modelled employing the simplified Byrne's formula, which relates the incremental volumetric strain $\left(\Delta \varepsilon_{v d}\right)$ to the cyclic shear and volumetric strains $(\gamma$ and $\varepsilon_{v d}$ ) as (Byrne 1991):

$$
\Delta \varepsilon_{v d}=C_{1} \gamma \exp \left[-C_{2}\left(\frac{\varepsilon_{v d}}{\gamma}\right)\right]
$$


where $C_{1}$ and $C_{2}$ are constants that in many cases are related to each other by: $C_{1} \cdot C_{2}=0.4$. $C_{1}$ can be calculated from the relative density $\left(\mathrm{D}_{\mathrm{r}}\right)$ as follows:

$$
C_{1}=7600\left(D_{r}\right)^{-2.5}
$$

The relative density in turn, may be defined as a function of the corrected SPT, $\left(\mathrm{N}_{1}\right)_{60}$,

$$
D_{r}=15 \sqrt{\left(N_{1}\right)_{60}}
$$

Another constant, $C_{3}$, is used in the model to define a threshold strain below which no excess pore pressure is generated. Following the calculation of the excess pore pressure, the program computes the effective stresses and applies them in the Mohr-Coulomb shear failure criterion.

\section{Experimental and numerical results}

Initially, experiment \#1 was performed on unfrozen saturated soil model to establish the mean shear wave velocity of the material, vital for realistic numerical modelling. To this end, the model was excited by the El Centro record and the natural frequency was estimated. The intensity of the original acceleration time history was scaled down to, $\mathrm{PGA}=0.05 \mathrm{~g}$, in order to ensure that the soil remains within the linear range. The obtained transfer function defined as the ratio of the Fourier amplitudes of the soil surface acceleration to those of the base motion acceleration. The obtained transfer function is displayed in Fig. 6.

Fig. 6: Transfer function of unfrozen soil deposit under low-intensity base excitation, PGA=0.05g. Dashed arrows indicate the first three natural frequencies of the model.

From the transfer function, the frequency corresponding to the first peak value was considered as the potential fundamental frequency $\left(\mathrm{f}_{1}\right)$ of the deposit. The natural frequencies of a soil layer can be approximated from (Kramer 1996):

$$
f_{n} \approx \frac{\bar{V}_{s}}{4 H}(2 n-1) \quad n=1,2,3, \ldots, \infty
$$

where $\bar{V}_{S}$ is the average shear wave velocity, $\mathrm{H}$ is the thickness of deposit and $\mathrm{n}$ is the mode number. Considering $\mathrm{f}_{1}=26 \mathrm{~Hz}$ and substituting $\mathrm{n}=1$ and $\mathrm{H}=0.4 \mathrm{~m}$ in the formula, the average shear wave velocity was estimated to be $42 \mathrm{~m} / \mathrm{sec}$. In order to examine the accuracy, two consecutive higher 
frequencies that were in the range of the input frequencies were also approximated by the formula $\left(\mathrm{f}_{2}=78 \mathrm{~Hz}\right.$ and $\mathrm{f}_{3}=130 \mathrm{~Hz}$ ) and are indicated in Fig. 6 by dashed arrows. As it can be observed from Fig. 6, they coincide with the global and local peaks of the transfer function. This confirms the validity of the computed average shear wave velocity.

The variation of soil stiffness along the soil profile considered in the numerical model was assumed to be parabolic based on the empirical equations for $\mathrm{G}_{\max }$ of sand (Seed and Idriss 1970). The distribution function was determined by trial and error such that its average value is close to the value back-figured from the experiment. This is accomplished by varying the stiffness distribution and comparing the calculated response with the measurements from the physical model test until the best match is achieved. The minimized error function was indeed the difference of the acceleration response spectra (SA) of the soil surface motion obtained from the numerical and experimental models. Fig. 7 demonstrates the best match of the responses after performing heightwise stiffness corrections. It should be noted that the spectra presented in this study are derived from the ground motions converted to the original time scales.

Fig. 7: Experimental and numerical acceleration response spectra after performing vertical stiffness corrections. In experiment \#2, a SCM block with thickness of $0.5 \mathrm{H}(20 \mathrm{~cm})$ was buried in the centre of the model leaving $1.25 \mathrm{~cm}$ of unfrozen soil above the block. The results of this test under the high level of excitation (PGA=0.5g) are shown in Fig. 8. It can be seen from Fig. 8a that the recorded response at the top of the frozen block is higher than that of the unfrozen soil for the period range of $0.2-5 \mathrm{sec}$. This is confirmed by the ratio of both responses displayed in Fig. 8b, which shows that the spectral response at top of the frozen block can be up to $60 \%$ higher than the response of the unfrozen soil.

Fig. 8: Results of experiment \#2. (a) Acceleration response spectra and (b) ratio of the frozen block and unfrozen soil response spectra.

Effect of block thickness: The effect of the frozen block thickness on the site response was investigated in experiments \#3, \#4 and \#5 with block heights of $0.25 \mathrm{H}(10 \mathrm{~cm}), 0.5 \mathrm{H}(20 \mathrm{~cm})$ and $\mathrm{H}$ $(40 \mathrm{~cm})$ as per Table 5 . In each test, two blocks were placed at a distance equal to five times the 
block width $(50 \mathrm{~cm})$. The unfrozen soil thickness remained constant and equal to $40 \mathrm{~cm}$. The results of the three tests under the high level of shaking intensity, i.e., PGA=0.5g, are illustrated in Fig. 9. Only minor differences in the responses of frozen and unfrozen soils were observed, thus suggesting that the relative thickness of the frozen blocks has insignificant effect on the site response.

Fig. 9: Acceleration response spectra at the top of (a) frozen blocks, and (b) unfrozen soil. The legends indicate the thickness of the frozen blocks, where $\mathrm{H}$ denotes the total thickness of the soil layers.

Effect of distance between frozen blocks: Two of the experiments, \#4 and \#6 (Table 5) were conducted to investigate the effect of the distance separating the frozen blocks (or span), $\mathrm{W}_{\mathrm{u}}$, on their interaction with unfrozen soil. Figs. 10a and b compare the experimental and numerical spectral acceleration responses obtained at the top of the model for experiments \#4 and \#6. For both tests, favourable agreement can be observed between the experimental and numerical response spectra (SA). In addition, the excess pore-water pressure ratio, $r_{u}$, was obtained at a depth of $5 \mathrm{~cm}$ within the unfrozen soil between the blocks. The excess pore-pressure ratio is defined as:

$$
r_{u}=\frac{\Delta u}{\sigma_{v}^{\prime}}
$$

where $\Delta u$ is the excess of pore water pressure and $\sigma_{v}^{\prime}$ is the vertical effective stress. Figs. 10c and d compare the experimental and numerical $r_{u}$ values obtained at a depth of $5 \mathrm{~cm}$ within the unfrozen soil between the blocks. Again, favourable agreement can be observed between the experimental and numerical results.

Theoretically, when the excess pore water pressure reaches the value of the vertical effective stress, i.e., $r_{u}=1$, the soil particles lose their contact and liquefaction occurs. No evidence of liquefaction, such as large displacements at the surface, could be observed in both experiments under the high level of shaking intensity (PGA=0.5g), but at the end of the vibrations the unfrozen soil seemed to be on the verge of liquefaction with relatively high $r_{u}\left(0.7<r_{u}<0.9\right)$.

Fig. 10: Spectral acceleration obtained from shaking table experiments (Exp) and numerical modelling (Num) for (a) $\mathrm{W}_{\mathrm{u}}=5 \mathrm{~W}_{\mathrm{f}}$ and (b) $\mathrm{W}_{\mathrm{u}}=3 \mathrm{~W}_{\mathrm{f}}$. Time histories of $\mathrm{r}_{\mathrm{u}}$ in unfrozen soils measured at depth of $5 \mathrm{~cm}$ at mid-distance between blocks for (c) $\mathrm{W}_{\mathrm{u}}=5 \mathrm{~W}_{\mathrm{f}}$ and (d) $\mathrm{W}_{\mathrm{u}}=3 \mathrm{~W}_{\mathrm{f}}$. $\mathrm{f}$ and $\mathrm{u}$ indicate frozen and unfrozen soil responses. 
The verified numerical model was used to predict soil behaviour for other distance combinations of the frozen soil blocks. The results of the parametric study for frozen blocks and unfrozen soil are depicted in Fig. 11. The spectral responses of frozen blocks remain almost constant whereas those of unfrozen soils generally decrease by increasing the span, $\mathrm{W}_{\mathrm{u}}$. The highest reduction for unfrozen soils is $36 \%$ and is observed at the peak spectral acceleration. Similar reduction was observed in PGA as well. In summary, $\mathrm{W}_{\mathrm{u}}$ has higher influence on the dynamic response of the unfrozen soils rather than that of the frozen blocks.

Theoretically, when the span approaches infinity, the spectral response of the unfrozen soil between the frozen block should reach the values of the unfrozen soil obtained in experiment \#1, and response of the frozen soil should attain the site response of a single block (experiment \#2). To check the results, the two mentioned boundary cases are also depicted by dashed lines in Fig. 11. In both cases, the dashed lines reside below the continuous curves and indicating that the numerical model predicts the trends correctly.

Fig. 11: Study of the effect of span length $\left(W_{u}\right)$ on site response in (a) frozen and (b) unfrozen soils.

Effect of block width: The parametric study results of the effect of block width $\left(\mathrm{W}_{\mathrm{f}}\right)$ on the site response are presented in Fig. 12. The span $\left(\mathrm{W}_{\mathrm{u}}\right)$ remained constant and equal to $50 \mathrm{~cm}$. As it can be noted from Fig. 12, the frozen soil response is considerably more sensitive to $W_{\mathrm{f}}$ than the unfrozen soil. The highest spectral accelerations of the frozen soil are obtained for the smallest widths $\left(\mathrm{W}_{\mathrm{f}}<0.2 \mathrm{~W}_{\mathrm{u}}\right)$. It seems that the shear stiffness of the frozen blocks, which is proportional to their width, plays a major role in their dynamic interaction with unfrozen soil. With the increase of $\mathrm{W}_{\mathrm{f}}$, the response decreases and becomes almost constant beyond $\mathrm{W}_{\mathrm{f}}=0.6 \mathrm{~W}_{\mathrm{u}}$. For the considered widths, the maximal difference between the peak spectral accelerations was $33 \%$ in frozen soil, compared to the only $5 \%$ decrease in unfrozen soil. Similar ratios were obtained for the PGA values.

Fig. 12: Study of the effect of block width $\left(\mathrm{W}_{\mathrm{f}}\right)$ on site response in (a) frozen and (b) unfrozen parts.

Effect of number of blocks (numerical simulations only): In the above lab experiments and accompanying numerical models, a maximum of two frozen blocks were considered. In the field, 
however, the intermittent character of the discontinuous permafrost can contribute to frequent occurrence of the frozen and unfrozen areas. To investigate the interaction when more than two frozen blocks are present, numerical simulations were performed in which the number of blocks was gradually increased from two to five while the distance between the blocks (span) remained constant $(50 \mathrm{~cm})$. The response of all the considered frozen blocks and the unfrozen soil between them was calculated. Since the results displayed low scattering and no specific trend could be observed in the responses, the minimum and maximum envelopes are shown in Fig. 13. The maximal differences between the peak spectral acceleration were in the order of $10 \%$ for both frozen blocks and unfrozen soil indicating that the soil response is not sensitive to the number of the considered frozen blocks when it is higher than two.

Fig. 13: Envelopes of the maximum and minimum spectral response for the number of frozen blocks varying between

2 and 5 . The distance between the blocks remained constant, $\mathrm{W}_{\mathrm{u}}=50 \mathrm{~cm}$.
Fig. 14: Sensitivity of the site response to varying shear wave velocity of the frozen blocks. The distance between the blocks remained constant, $\mathrm{W}_{\mathrm{u}}=50 \mathrm{~cm}$.

Sensitivity to shear wave velocity (numerical simulations only): As discussed in Section 2.3, the shear wave velocity of frozen sand and silty sand varies in a broad range of $900-2500 \mathrm{~m} / \mathrm{sec}$. In the above experiments (SCM blocks) and numerical simulations, $\mathrm{V}_{\mathrm{s}}$ of frozen soil was assumed equal to $1500 \mathrm{~m} / \mathrm{sec}$. To examine the sensitivity of the site response to the shear wave velocity of the frozen soils, a series of numerical analyses was performed in which $\mathrm{V}_{\mathrm{s}}$ was gradually increased from $1000 \mathrm{~m} / \mathrm{sec}$ to $2500 \mathrm{~m} / \mathrm{sec}$. In Fig. 14, only the results of the two extreme cases are presented with the other results falling between them. As can be observed in Fig. 14, the response of the frozen soil shows a descending trend with increase of the shear wave velocity, with a maximal difference of about $12 \%$ for the peak spectral response. At the same time, spectral response unfrozen soil remains almost constant.

Intensity of base excitation (laboratory experiments only): The presented experimental results above were obtained under the "high" base excitation. Most of the experiments were also repeated for different base excitations referred to as "low" (PGA=0.15g), "medium" (PGA=0.3g) and "high" 
$(\mathrm{PGA}=0.5 \mathrm{~g})$. To investigate the impact of the different levels of seismic excitation on the site response, the measured PGA values at the ground surface during all conducted shaking table experiments are presented in Fig. 15 for all the considered frozen block configurations. As expected, the obtained ground response of the frozen soil is systematically higher than that of the unfrozen soil for all three levels of seismic excitation. This is clearly demonstrated by comparing the average PGA response of the frozen and unfrozen soils indicated with dashed lines in Fig. 15. The ratio between the average PGAs of the frozen to unfrozen soils increased from about 1.25 for low intensity, to 1.30 for medium intensity and to 1.42 for high intensity earthquakes.

Fig. 15: Experimental readings of the PGA in frozen blocks and unfrozen soils for (a) low, (b) medium and (c) high intensities of base excitations. Dashed lines depict average of the PGAs.

Interaction of nonparallel blocks (laboratory experiments only): All experiments studied in this section were planned in a way that conditions of plane strain were satisfied. To investigate a general case in which the frozen blocks are not perpendicular to the direction of the input motion and are not parallel to each other (3D conditions), an additional experiment was conducted (experiment \#8) with a test setup as shown in Fig. 16. The distance between the frozen blocks varies from $10 \mathrm{~cm}$ to $50 \mathrm{~cm}$ with an average of $30 \mathrm{~cm}$ at the centre of the blocks.

Fig. 16: Top view of the test setup of experiment \#8. Dashed lines represent the location of blocks in experiment \#7 for comparison.

To determine the effect of the direction of the frozen blocks on the site response motion, the acceleration of the unfrozen soil was recorded at the mid-distance between the frozen blocks by using a biaxial accelerometer. Under "low", "medium" and "high" excitation levels, the transversal PGAs were $13.3,13.1$ and $12.6 \%$ of the longitudinal ones respectively. Furthermore, the ground responses were compared to those of a plane strain case in which the unfrozen span was $30 \mathrm{~cm}$, i.e., equal to the average of the current variable span. The specifications of the plane strain case are presented in Table 5 (experiment \#7) and the corresponding block layout is shown by dashed lines in Fig. 16. The results of the two experiments are presented in Fig. 17. As it can be seen, the spectra 
derived from both experiments agree well for both frozen and unfrozen soils. Also, PGAs of frozen blocks and unfrozen soil show 4.1 and 2.5\% variations respectively. The results of Experiments \#7 and \#8 demonstrate that the response of the 3D case can be approximated by the response of the simplified 2D plane strain model with reasonable accuracy.

Fig. 17: Comparison of the free-field responses of plane strain and 3D cases in (a) frozen and (b) unfrozen soils.

\section{Practical application of the results}

Performing controlled lab experiments fully satisfying the physical aspects of a complex phenomenon such as permafrost in many cases, is theoretically and technically impossible. Therefore, making some assumptions and simplifications is inevitable. For example, the shear wave velocity of the permafrost is a temperature-dependent parameter. Since the permafrost is subjected to a vertical temperature gradient, certain variation of the shear wave velocity with depth can be expected. LeBlanc et al. (2004) studied cryostratigraphy of a permafrost near Umiujaq in northern Quebec, Canada, performing seismic cone penetration and tomography tests. Based on their results typical temperature and $V_{s}$ schematic profiles are illustrated in Fig. 18. As it can be seen, below a specific depth where the temperature approaches zero, degradation of $V_{s}$ starts. Between top of permafrost and this depth $V_{s}$ has its largest values and smallest fluctuations and can be replaced by an average constant value as shown in Fig. 18. Since below the effective depth $V_{s}$ of the frozen soil tends to that of the adjacent unfrozen soil, dynamic interaction between them reaches to its minimum, particularly at higher depths. Neglecting the active layer and simulating the effective depth of permafrost only was therefore a reasonable approximation in the experiments. For practical applications and according to the local temperature gradient an appropriate effective depth should be selected as thickness of the frozen block $(\mathrm{H})$.

Fig. 18: Schematic profile of temperature and shear wave velocity $\left(V_{s}\right)$ in depth of permafrost based on LeBlanc et al. (2004).

In discontinuous permafrost regions, soil conditions change spatially from frozen to unfrozen and vice versa. A schematic presentation of such a transition zone over which shear wave velocity 
gradually changes is given in Fig. 19. Due to practical difficulties such transitions could not be simulated in the experiments and the obtained results correspond to cases with abrupt changes of shear wave velocity. This simplification affects the magnitude of the measured parameters. To make the laboratory results transferable to real field conditions, the lateral interaction of frozen and unfrozen soils has to be considered. Due to the considerably higher shear wave velocity, frozen soil acts like an embedded vertical shear beam that affects the site response by having interaction with unfrozen soil. According to this simple shear model, at any depth permafrost and transition zones can be treated as parallel springs with different stiffness factors. Thus, assuming the shear stiffness at any depth as proportional to the product of the shear modulus and the cross-sectional area of the frozen block, one can account for the effects of the transition zones by modifying the shear stiffness of permafrost. The total stiffness of such a frozen block system of permafrost and transition zones is:

$$
K_{f}=K_{p}+K_{t}
$$

where $K_{f}, K_{p}$ and $K_{t}$ are the stiffness factors of the frozen block system, permafrost and the transition zones, respectively. Since the shear modulus is proportional to the square of shear wave velocity, in a deposit with unit thickness (cross-sectional area=width) eq. (8) can be written as:

$$
W_{f} V_{s}^{2}=W_{p} V_{s p}^{2}+W_{t} V_{s t}^{2}
$$

where $\mathrm{W}_{\mathrm{f}}$ is the total width of the frozen block system, $\mathrm{W}_{\mathrm{p}}$ is the width of the permafrost, $\mathrm{W}_{\mathrm{t}}$ is the total width of the transition zones and, $V_{\text {sp }}$ and $V_{\text {st }}$ are the corresponding average shear wave velocities. According to the results presented in Section 4, an increase of the lateral shear stiffness of the frozen block by increasing either $\mathrm{V}_{\mathrm{s}}$ or $\mathrm{W}_{\mathrm{f}}$, leads to decrease of frozen block response (Figs. 12 and 14). However, this does not affect the unfrozen soil response. Therefore, response of the frozen block is conservatively higher if the effect of transition zones is neglected.

Fig. 19: Schematic horizontal distribution of temperature and shear wave velocity in discontinuous permafrost regions and spring model of permafrost-transition zones system. 
Another parameter to be considered is presence of some unfrozen water in permafrost and its potential viscoelastic effects on energy dissipation. Two major damping mechanisms should be considered in wave propagation problems: hysteretic and viscous. The former is strain-dependent and proportional to the level of nonlinearity that the material experiences and the latter is frequency-dependent and increases with increase of wave frequency. In the case of seismic site response analysis, strain levels in the prototype are high and frequency levels are low. Thus, the damping is predominantly hysteretic rather than viscous. However this is not the case in a model. As discussed earlier, the simulation rule for the stiffness of the frozen material $\left(\mathrm{V}_{s}\right)$ was satisfied in the model. However, shear strength of the blocks were not scaled down properly and the sandcement mixture had some over strength. Therefore, the blocks had a wider linear range and the generated hysteretic damping was smaller than anticipated. On the other hand, according to the simulation rules, the frequency content of the dynamic loading was scaled up (increased 32 times and up to $150 \mathrm{~Hz}$ ) and consequently, the contribution of the viscous damping in energy dissipation in the model was higher when compared to the prototype. This suggests that the model was still subjected to a reasonable amount of damping despite the different predominant damping mechanism compared to the prototype.

\section{Conclusions}

Laboratory experiments and numerical simulations were conducted to investigate the site effects in discontinuous permafrost conditions characterised with the intermittent presence of frozen soils. Particular attention was given to the dynamic interaction between the frozen soil blocks with the surrounding unfrozen soil. A series of shaking table tests with small-scale physical models was designed to conduct the experiments and to provide data to validate the numerical FLAC models. The following conclusions are drawn from this study:

- The obtained spectral response of the frozen soils is systematically higher than that of the unfrozen soils. 
- The distance between the frozen blocks had a notable influence on the response of unfrozen soil, whereas frozen block responses were less sensitive. The spectral acceleration values of unfrozen soil generally decreased by increasing the distance.

- The unfrozen soil response was not sensitive to the width of the frozen blocks. At the same time, the frozen block response showed a decreasing trend (about 30\%) for a 10 fold increased width.

- The relative thickness of the frozen blocks was not an important parameter in both of frozen and unfrozen soil response.

- Including more than two frozen blocks did not contribute to any observable trend in the soil responses. The envelopes of the minimum and maximum spectral accelerations showed about $10 \%$ differences for both frozen blocks and unfrozen soils.

- The increase of the shear wave velocity of the frozen blocks from $1000 \mathrm{~m} / \mathrm{sec}$ to $2500 \mathrm{~m} / \mathrm{sec}$ contributed to a decrease of the response spectral accelerations of the frozen blocks of about $12 \%$. At the same time, the response of the unfrozen soils remained fairly constant.

- Free-field response of a physical model in which plane strain conditions were violated (with nonparallel blocks) was successfully simulated by a simplified 2D plane strain model in the lab. The experiments also further revealed that the perpendicular-to-excitation component of the free-field response is small compared to the parallel-to-excitation component.

The simplified physical model along with the numerical model used in this research generally addressed the phenomenon and revealed some significant pieces of information regarding the seismic site response of regions with discontinuous permafrost. Site investigations and monitoring are required to examine the reliability of the achieved results and to discover further aspects of the problem. 


\section{Acknowledgements}

Funding was provided in part through the Canadian Safety and Security Program (CSSP) led by

Defence Research and Development Canada's Centre for Security Science, in partnership with Public Safety Canada. Additional support was provided by the Natural Sciences and Engineering Research Council of Canada (NSERC) as grant in-aid to second author.

\section{References}

Alam M. J., and Towhata, I. 2008. Some important aspects of physical modelling of liquefaction in 1g shaking table. 2008 Seismic Engineering Conference: Commemorating the 1908 Messina and Reggio Calabria Earthquake, Reggio Calabria, Italy, 8-11 July 2008. American Institute of Physics.

Bishop, A.W., Green, G.E., Garga, V.K., Andresen, A., and Brown, J.D. 1971. A new ring shear apparatus and its application to the measurement of residual strength. Geotechnique, 21(4), 273-328.

Byrne, P. 1991. A cyclic shear-volume coupling and pore-pressure model for sand. In Second International Conference on Recent Advances in Geotechnical Earthquake Engineering and Soil Dynamics, St. Louis, Mo., 11-15 March 1991.

Cheng, N. 2008. Formula for the viscosity of a Glycerol-water mixture. Industrial and Engineering Chemistry Research, 47: 3285-3288.

Electric Power Research Institute. 1993. Guidelines for determining design basic ground motions. Volume 1. Electric Power Research Institute.

Finn, W. D. L., and Yong, R. N. 1978. Seismic response of frozen ground. Journal of the Geotechnical Engineering Division, 104(10): 1225-1241. 
Finn, W. D. L., Yong, R. N., and Lee, K. W. 1978. Liquefaction of thawed layers in frozen soils. Journal of the Geotechnical Engineering Division, 104(10): 1243-1255.

Itasca Consulting Group, Inc. 2002. FLAC, fast lagrangian analysis of continua, user's guide. Minneapolis, Minn.

Hardin, B. O., and Drnevich, V. P. 1972. Shear modulus and damping in soils: Design equations and curves. Journal of Soil Mechanics and Foundations Division, 98(7): 667-692.

Hashash, Y. M. A., Phillips, C., and Groholski, D. R. 2010. Recent advances in non-linear site response analysis. In Fifth International Conference on Recent Advances in Geotechnical Earthquake Engineering and Soil Dynamics, San Diego, Calif., 24-29 May 2010.

Iai, S. 1988. Similitude for shaking table tests on soil-structure-fluid model in $1 \mathrm{~g}$ gravitational field. Yokosuka: The Port and Harbour Research Institute.

Iwasaki, T., Tatsuoka, F., and Takagi., Y. 1978. Shear modulus of sands under torsional shear loading. Soils and Foundations, 18(1): 39-56.

Johnston, G. H. 1981. Permafrost: Engineering, design and construction. John Wiley \& Sons, Toronto.

Kline, S. 1965. Similitude and approximation theory. McGraw-Hill, New York, N.Y.

Kokusho, T. 1980. Cyclic triaxial test of dynamic soil properties for wide strain range. Soils and Foundations, 20(2): 45-60.

Kramer, S. L. 1996. Geotechnical earthquake engineering. Prentice Hall, Upper Saddle River, N.J.

LeBlanc, A. M., Fortier, R., Allard, M., Cosma, C., and Buteau, S. 2004. Seismic cone penetration test and seismic tomography in permafrost. Canadian Geotechnical Journal, 41: 796-813. 
Martin, G. R., Finn, W. D. L., and Seed, H. B. 1975. Fundamentals of liquefaction under cyclic loading. Journal of the Geotechnical Engineering Division, 101(5): 423-438.

Moncarz, P., and Krawinkler, H. 1981. Theory and application of experimental model analysis in earthquake engineering. Report No. 50, John A. Blume Earthquake Engineering Center, Stanford University, Stanford, Calif.

Muir Wood, D. 2004. Geotechnical modelling. Spon Press, New York, N.Y.

Muller, S. W. 2008. Frozen in time: Permafrost and engineering problems. ASCE, Reston, Va.

Nakano, Y., and Froula, N. H. 1973. Sound and shock transmission in frozen soils. In 2nd International Conference on Permafrost, Washington, D.C.

Qi, J., Ma, W., and Song, C. 2008. Influence of freeze-thaw on engineering properties of a silty soil. Cold Regions Science and Technology, 53: 397-404.

Rocha, M. 1957. The possibility of solving soil mechanics problems by use of models. In Proceedings of the Fourth International Conference on Soil Mechanics and Foundation Engineering, London, U.K., 12-24 August 1957, London, pp. 183-188.

Roscoe, K. 1968. Soils and model tests. Journal of Strain Analysis, 3(1): 57-64.

Seed, H. B., and Idriss, I. M. 1970. Soil moduli and damping factors for dynamic response analyses. Earthquake Engineering Research Center, Berkeley, Calif.

Singh, S., and Donovan, N. C. 1977. Seismic response of frozen-thawed soil systems. In 6th International Conference on Earthquake Engineering, New Delhi.

Towhata, I. 2008. Geotechnical earthquake engineering. Springer-Verlag, Berlin. 
Tsytovich, N. A. 1975. The mechanics of frozen ground. McGraw-Hill Book Company, Washington, D.C.

Turan, A., Hinchberger, S. D., and El Naggar, H. 2009. Design and commissioning of a laminar soil container for use on small shaking tables. Soil Dynamics and Earthquake Engineering, 29: 404-414.

Vargas-Monge, W. 1998. Ring shear tests on large deformation of sand. Ph.D. thesis, University of Tokyo, Tokyo.

Verdugo, R., and Ishihara, K. 1996. The steady state of sandy soils. Soils and Foundations, 36(2): 81-91.

Viklander, P. 1998. Permeability and volume changes in till due to cyclic freeze/thaw. Canadian Geotechnical Journal, 35: 471-477.

Wang, L., Wu, Z., Sun, J., Liu, X., and Wang, Z. 2009. Characteristics of ground motion at permafrost sites along the Qinghai-Tibet railway. Soil Dynamics and Earthquake Engineering, 29: 974981.

Yang, Z., Dutta, U., Xu, G., Hazirbaba, K., and Marx, E. E. 2011. Numerical analysis of permafrost effects on the seismic site response. Soil Dynamics and Earthquake Engineering, 31: 282290.

Zhang, J., Andrus, R. D., and Juang, C. H. 2005. Normalized shear modulus and material damping ratio relationships. Journal of Geotechnical and Geoenvironmental Engineering, 131(4): 453-464.

Zimmerman, R. W., and King, M. S. 1986. The effect of the extent of freezing on velocities in unconsolidated permafrost. Geophysics, 51(6): 1285-1290. 
Geological Survey of Canada. Digital borehole geotechnical database for the Mackenzie Valley/Delta region [online]. Available from http://geogratis.gc.ca/api/en/nrcan-rncan/esssst/13a48ecb-e6e4-5dd1-84cc-078d9ddc0b87.html [cited 2014].

Yukon Geological Survey. Alaska Highway geotechnical borehole database [online]. Available from http://ygsftp.gov.yk.ca/YukonPermafrostNetwork/AK\%20HWY\%20borehole\%20DB.htm [cited 2014]. 


\section{Figure Captions}

Fig. 1: Dynamic interaction between frozen soil blocks (in gray color) and the surrounding unfrozen soil.

Fig. 2: Test setup including (from left to right): the data acquisition system, the electric control module, the shaking table with the mounted flexible container with 12 frames; and sand-cement blocks representing frozen soils.

Fig. 3: Particle size distribution curves.

Fig. 4: Schematic presentation of the typical test setup (a) longitudinal cross section and (b) top view. Frozen soil blocks are indicated in grey color.

Fig. 5: Shear modulus reduction curves for frozen sand at $-1^{\circ} \mathrm{C}$ by Singh and Donovan (1977) and average unfrozen sand by Seed and Idriss (1970).

Fig. 6: Transfer function of unfrozen soil deposit under low-intensity base excitation, PGA=0.05g. Dashed arrows indicate the first three natural frequencies of the model.

Fig. 7: Experimental and numerical acceleration response spectra after performing vertical stiffness corrections.

Fig. 8: Results of experiment \#2. (a) Acceleration response spectra and (b) ratio of the frozen block and unfrozen soil response spectra.

Fig. 9: Acceleration response spectra at the top of (a) frozen blocks, and (b) unfrozen soil. The legends indicate the thickness of the frozen blocks, where $\mathrm{H}$ denotes the total thickness of the soil layers.

Fig. 10: Spectral acceleration obtained from shaking table experiments (Exp) and numerical modelling (Num) for (a) $\mathrm{W}_{\mathrm{u}}=5 \mathrm{~W}_{\mathrm{f}}$ and $(\mathrm{b}) \mathrm{W}_{\mathrm{u}}=3 \mathrm{~W}_{\mathrm{f}}$. Time histories of $\mathrm{r}_{\mathrm{u}}$ in unfrozen soils measured at depth of $5 \mathrm{~cm}$ at mid-distance between blocks for (c) $W_{u}=5 W_{f}$ and (d) $W_{u}=3 W_{f}$. f and $u$ indicate frozen and unfrozen soil responses. 
Fig. 11: Study of the effect of span length $\left(W_{u}\right)$ on site response in (a) frozen and (b) unfrozen soils.

Fig. 12: Study of the effect of block width $\left(\mathrm{W}_{\mathrm{f}}\right)$ on site response in (a) frozen and (b) unfrozen parts.

Fig. 13: Envelopes of the maximum and minimum spectral response for the number of frozen blocks varying between 2 and 5 . The distance between the blocks remained constant, $\mathrm{W}_{\mathrm{u}}=50 \mathrm{~cm}$.

Fig. 14: Sensitivity of the site response to varying shear wave velocity of the frozen blocks. The distance between the blocks remained constant, $\mathrm{W}_{\mathrm{u}}=50 \mathrm{~cm}$.

Fig. 15: Experimental readings of the PGA in frozen blocks and unfrozen soils for (a) low, (b) medium and (c) high intensities of base excitations. Dashed lines depict average of the PGAs.

Fig. 16: Top view of the test setup of experiment \#8. Dashed lines represent the location of blocks in experiment \#7 for comparison.

Fig. 17: Comparison of the free-field responses of plane strain and 3D cases in (a) frozen and (b) unfrozen soils.

Fig. 18: Schematic profile of temperature and shear wave velocity $\left(V_{s}\right)$ in depth of permafrost based on LeBlanc et al. (2004).

Fig. 19: Schematic horizontal distribution of temperature and shear wave velocity in discontinuous permafrost regions and spring model of permafrost-transition zones system. 


\section{Appendix: Technical specifications of measuring instruments}

Accelerometers: Make and model: Analog Devices ${ }^{\mathrm{TM}}$ (ADXL203 Dual-axis), Acceleration range: $\pm 5 g$, Specified Voltage: $5 \mathrm{~V}$, Operating temperature range: $-40{ }^{\circ} \mathrm{C}$ to $+125^{\circ} \mathrm{C}$, Maximum nonlinearity: \pm 1.25 , Dimensions (in water resistant shield): $22 \mathrm{~mm} \times 19 \mathrm{~mm} \times 15 \mathrm{~mm}$, Weight (including water resistant shield): $8.5 \mathrm{gr}$.

Pressure transducers: Make and model: Measurement Specialties ${ }^{\mathrm{TM}}$ (EPB-PW), Pressure range: $0.1 \mathrm{MPa}$, Full scale output (FSO): $30 \mathrm{mV}$, Operating temperature range: $-40^{\circ} \mathrm{C}$ to $+80^{\circ} \mathrm{C}$, Nonrepeatability: $\pm 0.25 \%$ FSO, Thermal zero shift: $\pm 4 \%$ FSO $/ 50{ }^{\circ} \mathrm{C}$, Thermal sensitivity shift: $\pm 2 \%$ FSO $/ 50{ }^{\circ} \mathrm{C}$, Dimensions (body): $\emptyset 6.4 \mathrm{~mm} \times 11.4 \mathrm{~mm}$, Weight (body): $0.8 \mathrm{gr}$. 


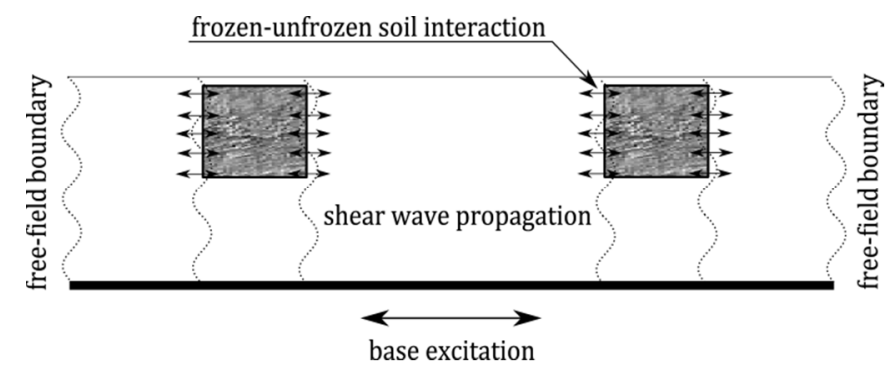

Fig. 1: Dynamic interaction between frozen soil blocks (in gray color) and the surrounding unfrozen soil.

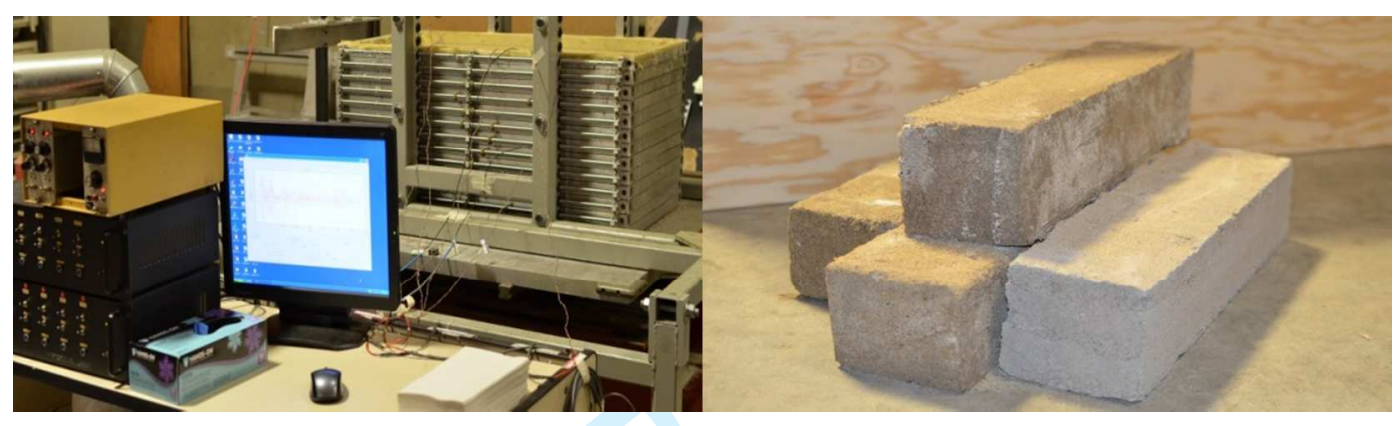

Fig. 2: Test setup including (from left to right): the data acquisition system, the electric control module, the shaking table with the mounted flexible container with 12 frames; and sand-cement blocks representing frozen soils.

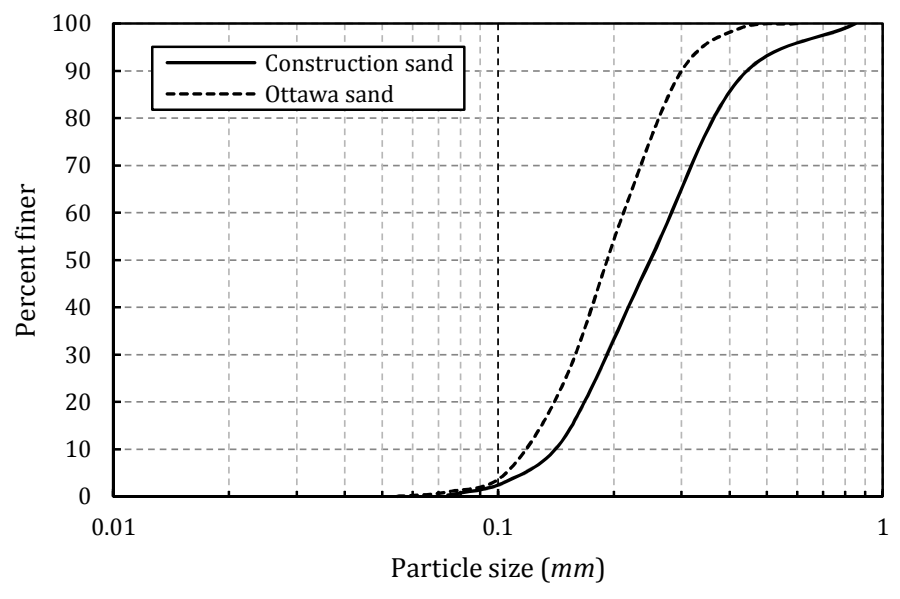

Fig. 3: Particle size distribution curves. 
(a) Longitudinal cross section

(b) Top view

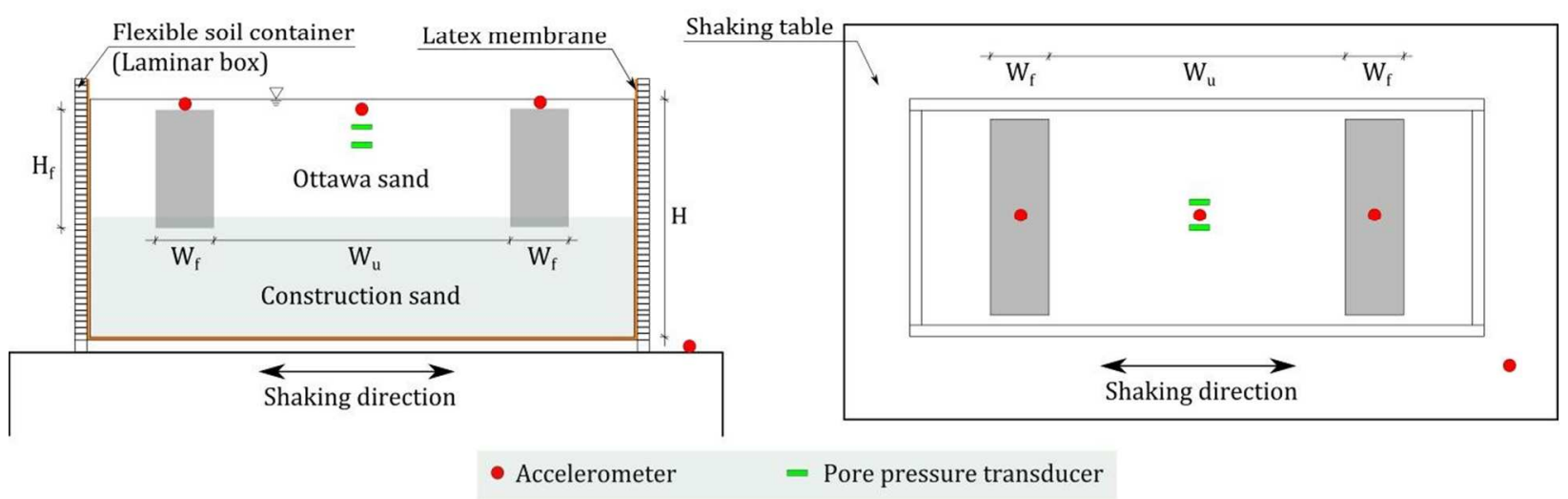

Fig. 4: Schematic presentation of the typical test setup (a) longitudinal cross section and (b) top view. Frozen soil blocks are indicated in grey color.

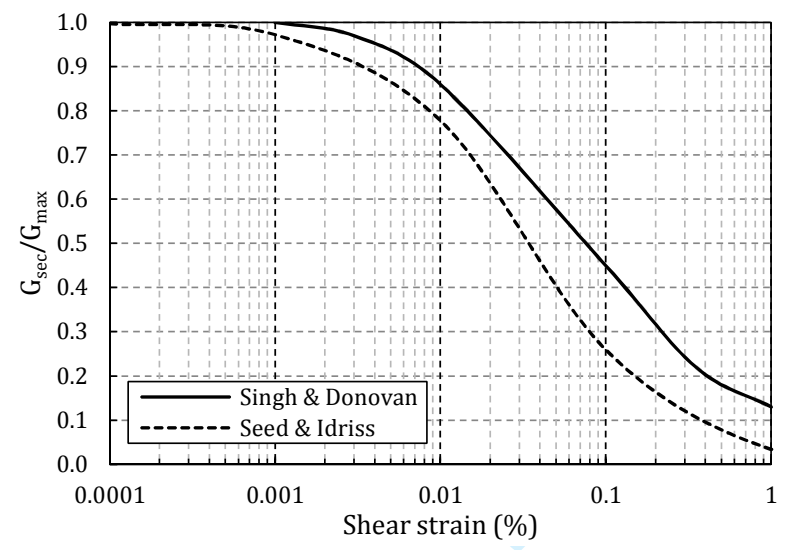

Fig. 5: Shear modulus reduction curves for frozen sand at $-1^{\circ} \mathrm{C}$ by Singh and Donovan (1977) and average unfrozen sand by Seed and Idriss (1970).

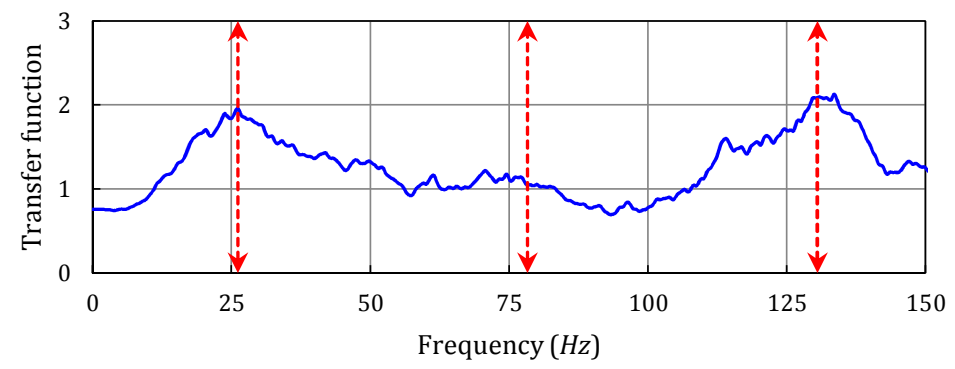

Fig. 6: Transfer function of unfrozen soil deposit under low-intensity base excitation, PGA=0.05g. Dashed arrows indicate the first three natural frequencies of the model. 


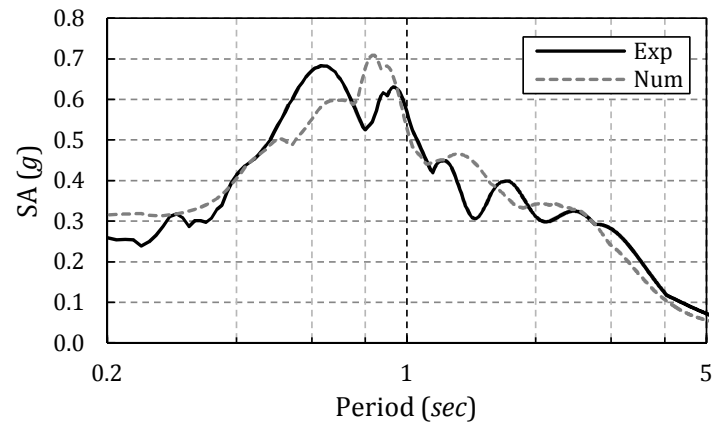

Fig. 7: Experimental and numerical acceleration response spectra after performing vertical stiffness corrections.

(a)

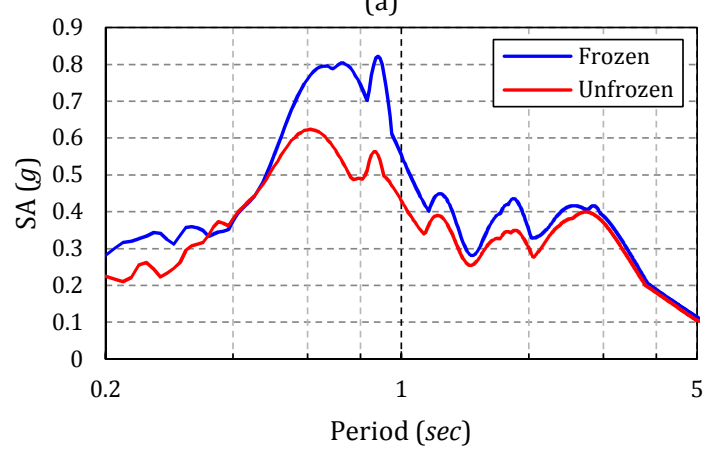

(b)

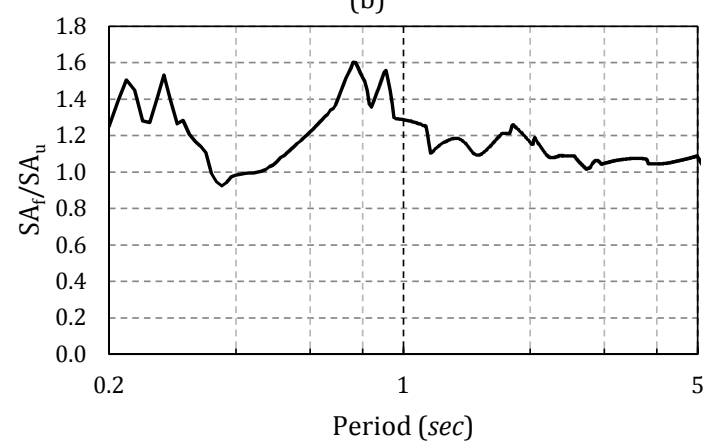

Fig. 8: Results of experiment \#2, (a) Acceleration response spectra and (b) ratio of the frozen block and unfrozen soil response spectra.

(a) Frozen block

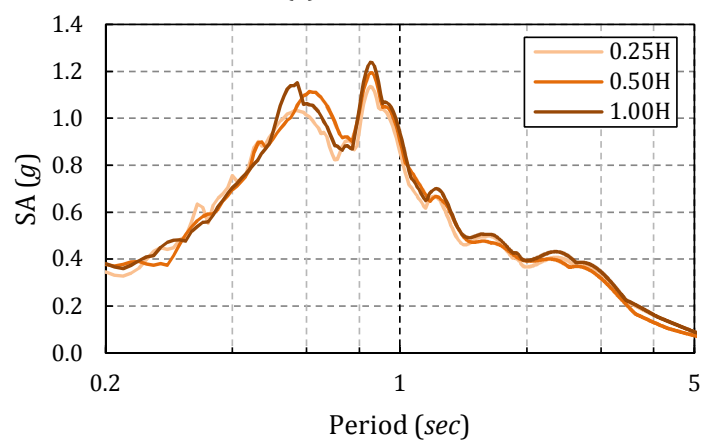

(b) Unfrozen soil

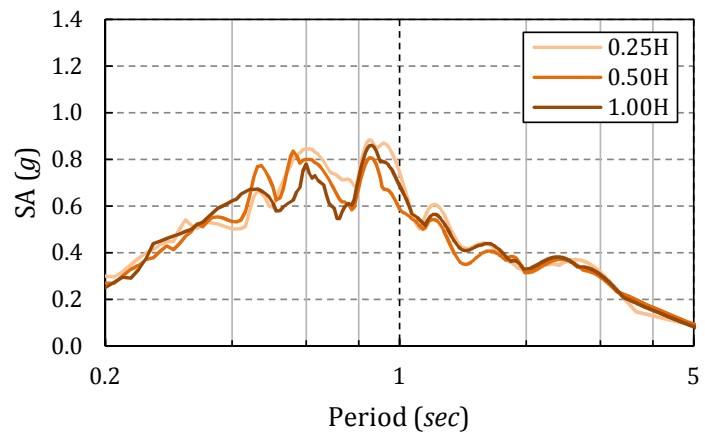

Fig. 9: Acceleration response spectra at the top of (a) frozen blocks, and (b) unfrozen soil. The legends indicate the thickness of the frozen blocks, where $\mathrm{H}$ denotes the total thickness of the soil layers. 
(a) $\mathrm{W}_{\mathrm{u}}=5 \mathrm{~W}_{\mathrm{f}}$

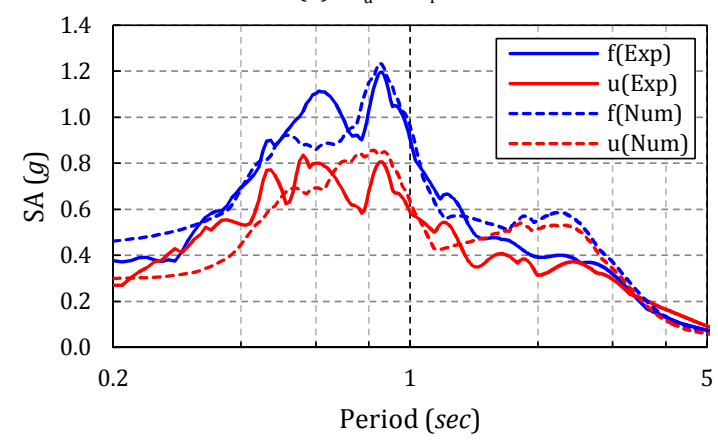

(c) $\mathrm{W}_{\mathrm{u}}=5 \mathrm{~W}_{\mathrm{f}}$

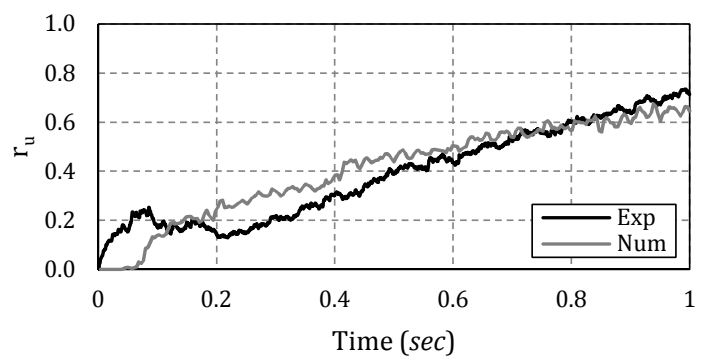

(b) $\mathrm{W}_{\mathrm{u}}=3 \mathrm{~W}_{\mathrm{f}}$

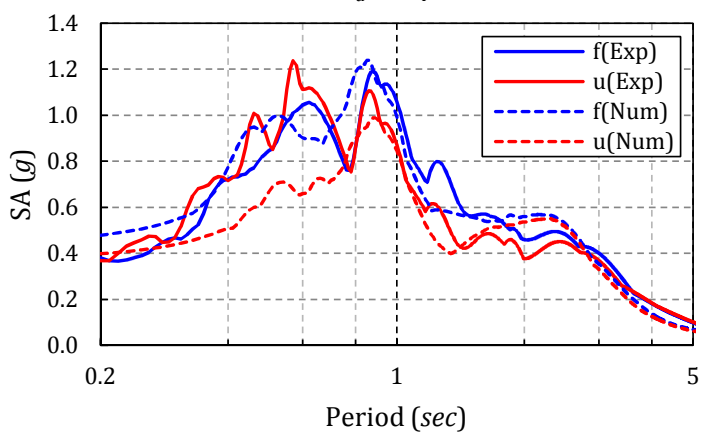

(d) $W_{u}=3 W_{f}$

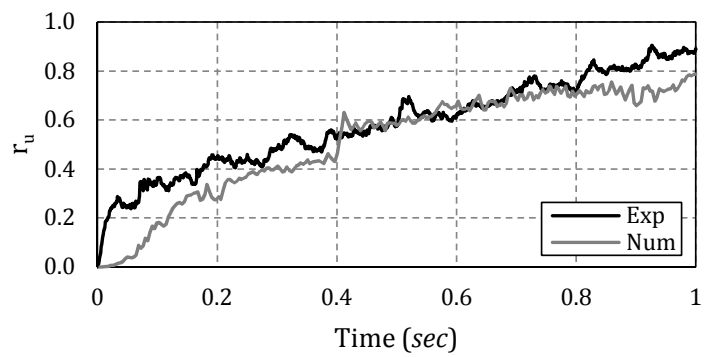

Fig. 10: Spectral acceleration obtained from shaking table experiments (Exp) and numerical modelling (Num) for (a) $\mathrm{W}_{\mathrm{u}}=5 \mathrm{~W}_{\mathrm{f}}$ and (b) $\mathrm{W}_{\mathrm{u}}=3 \mathrm{~W}_{\mathrm{f}}$. Time histories of $\mathrm{r}_{\mathrm{u}}$ in unfrozen soils measured at depth of $5 \mathrm{~cm}$ at mid-distance between blocks for (c) $\mathrm{W}_{\mathrm{u}}=5 \mathrm{~W}_{\mathrm{f}}$ and (d) $\mathrm{W}_{\mathrm{u}}=3 \mathrm{~W}_{\mathrm{f}}$. $\mathrm{f}$ and $\mathrm{u}$ indicate frozen and unfrozen soil responses.

(a) Frozen block

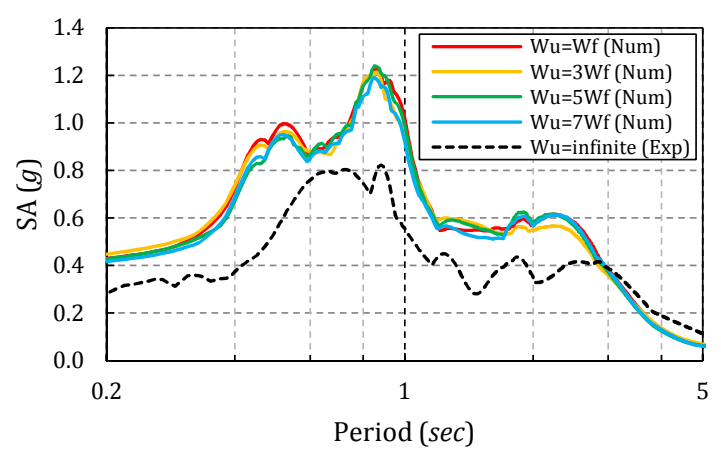

(b) Unfrozen soil

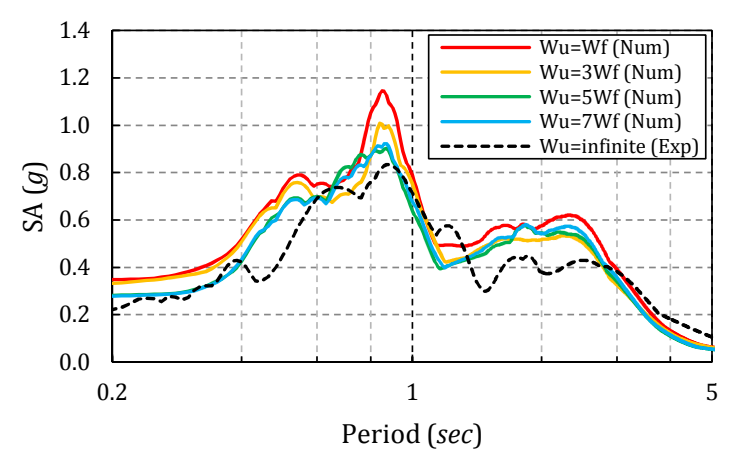

Fig. 11: Study of the effect of span length $\left(W_{u}\right)$ on site response in (a) frozen and (b) unfrozen soils. 
(a) Frozen block

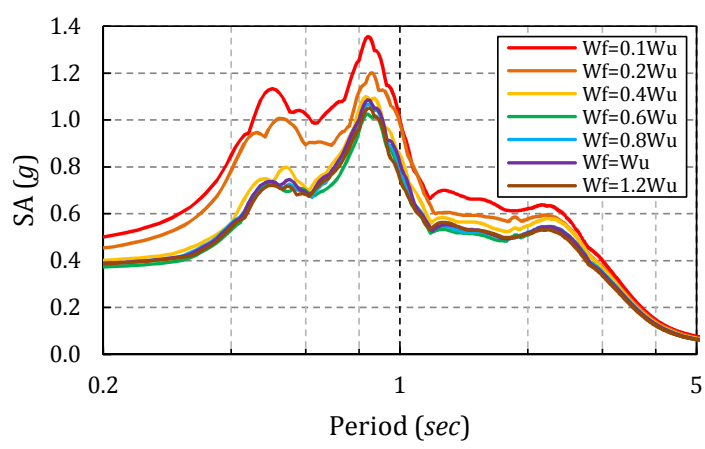

(b) Unfrozen soil

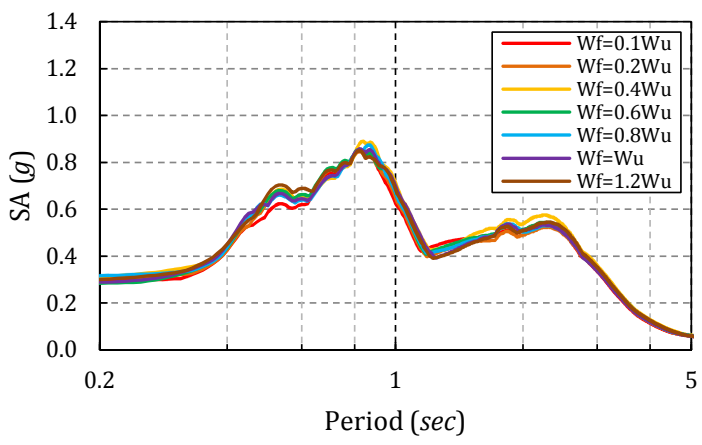

Fig. 12: Study of the effect of block length $\left(\mathrm{W}_{\mathrm{f}}\right)$ on site response in (a) frozen and (b) unfrozen soils.

Effect of number of blocks on response

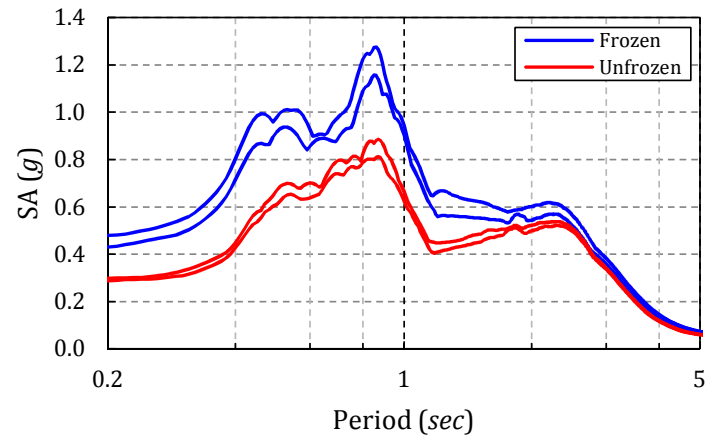

Fig. 13: Envelopes of the maximum and minimum spectral response for the number of frozen blocks varying between 2 and 5 . The distance between the blocks remained constant, $\mathrm{W}_{\mathrm{u}}=50 \mathrm{~cm}$.
Sensitivity of response to shear wave velocity

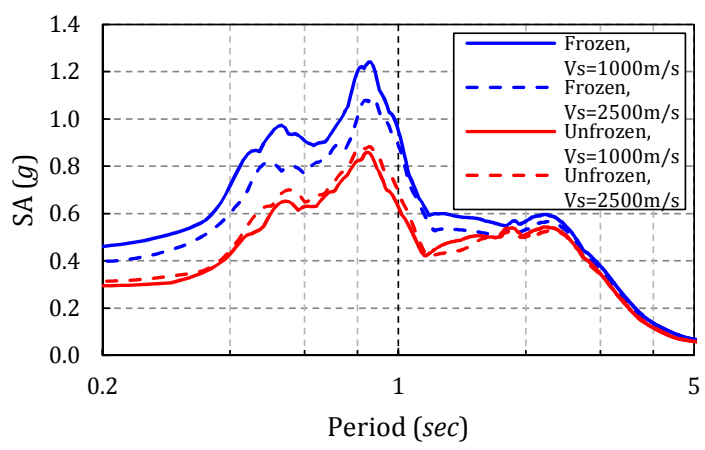

Fig. 14: Sensitivity of the site response to varying shear wave velocity of the frozen blocks. The distance between the blocks remained constant, $\mathrm{W}_{\mathrm{u}}=50 \mathrm{~cm}$. (a) Low intensity $(\mathrm{PGA}=0.15 \mathrm{~g})$

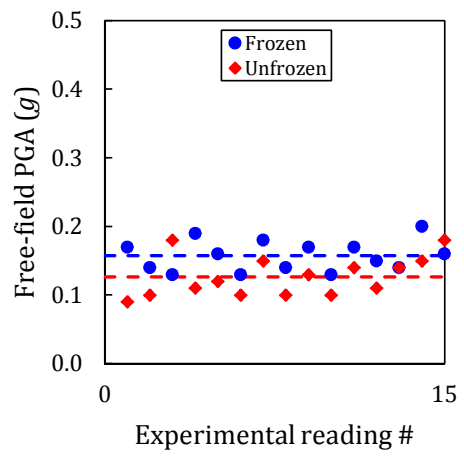

(b) Medium intensity ( $\mathrm{PGA}=0.3 \mathrm{~g}$ )

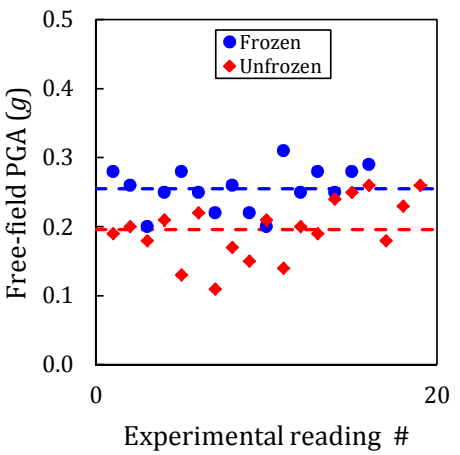

(c) High intensity (PGA=0.5g)

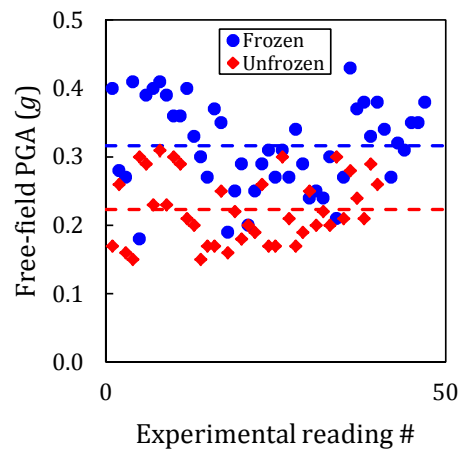

Fig. 15: Experimental readings of the PGA in frozen blocks and unfrozen soils for (a) low, (b) medium and (c) high intensities of base excitations. Dashed lines depict average of the PGAs. 


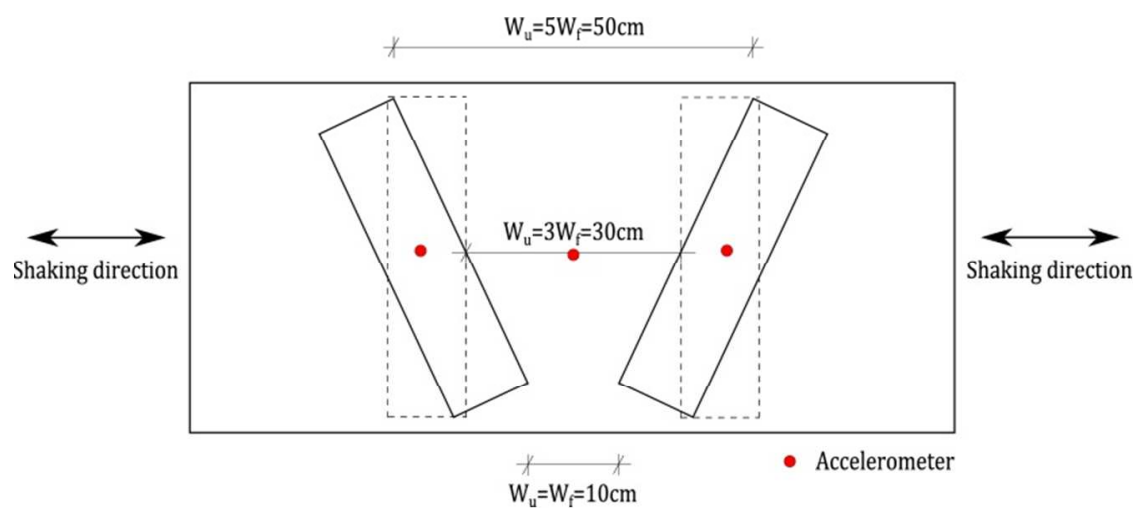

Fig. 16: Top view of the test setup of experiment \#8. Dashed lines represent the location of blocks in experiment \#7 for comparison.

(a) Frozen block

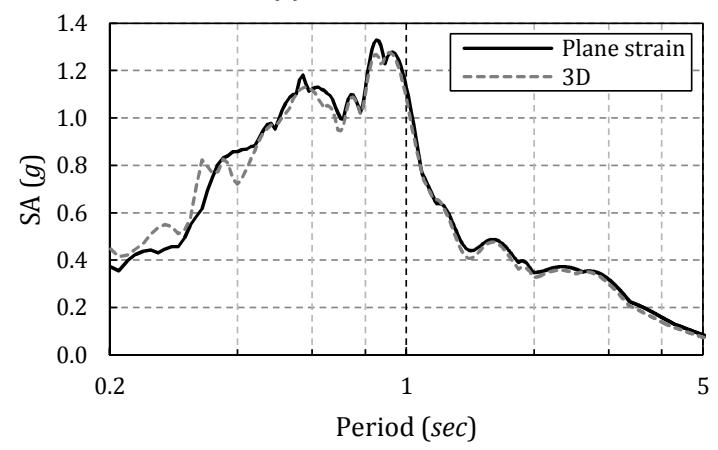

(b) Unfrozen soil

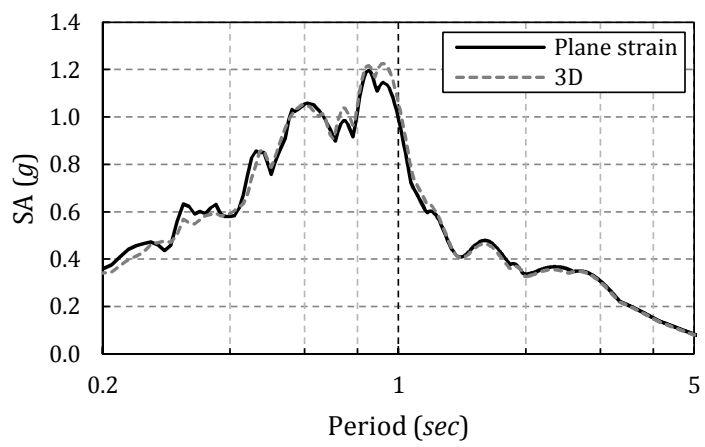

Fig. 17: Comparison of the free-field responses of plane strain and 3D cases in (a) frozen and (b) unfrozen soils. 


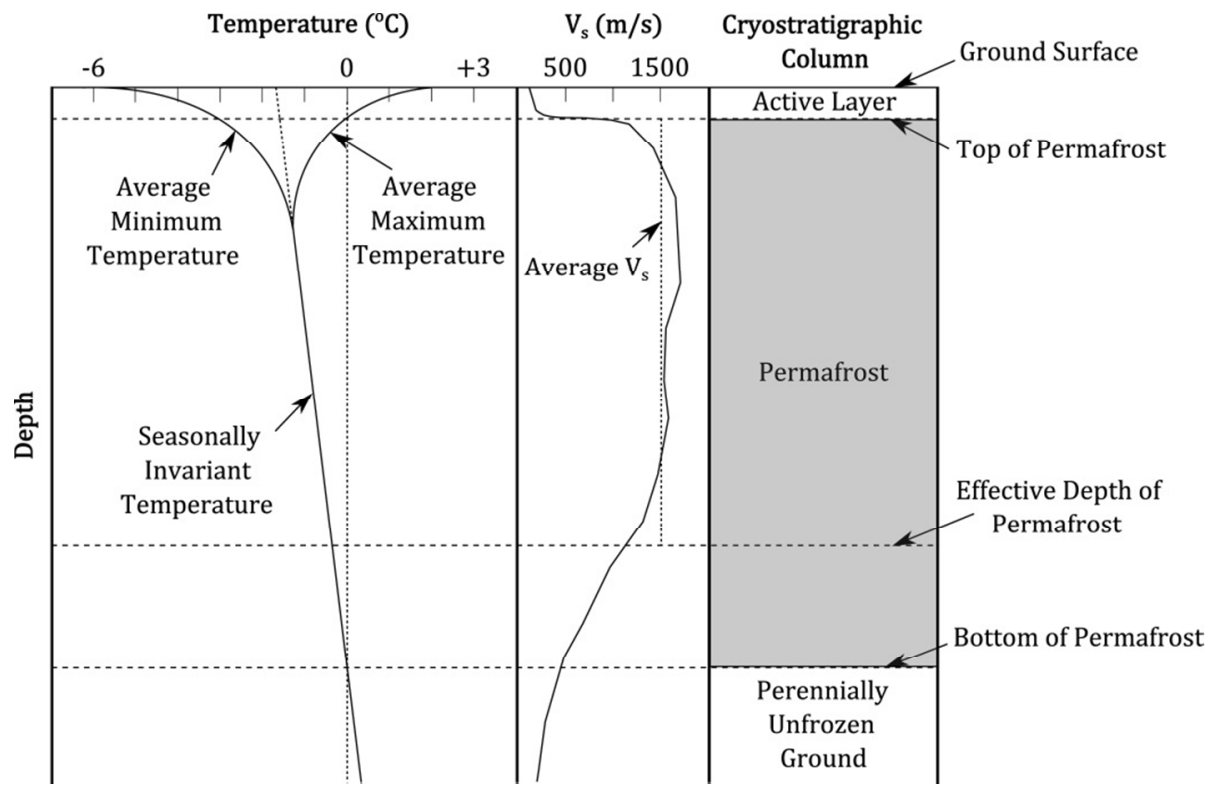

Fig. 18: Schematic profile of temperature and shear wave velocity $\left(V_{s}\right)$ in depth of permafrost based on LeBlanc et al. (2004).

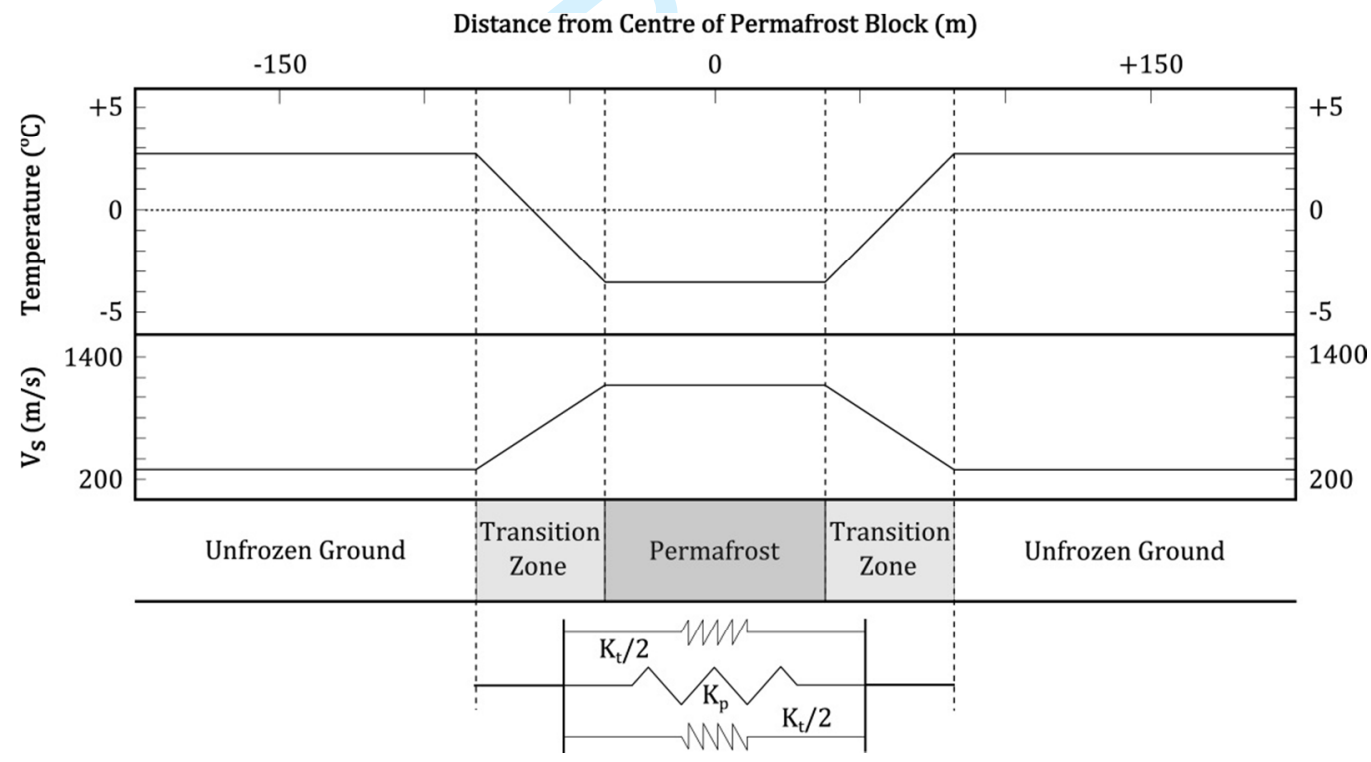

Fig. 19: Schematic horizontal distribution of temperature and shear wave velocity in discontinuous permafrost regions and spring model of permafrost-transition zones system. 
Table 1: Scale factors for $1 \mathrm{~g}$ shaking table tests on soil-structure-fluid models (Iai 1988).

\begin{tabular}{lcc}
\hline \multicolumn{1}{c}{ Item } & Scaling factor & Value \\
\hline Length & $\lambda_{\mathrm{L}}$ & 100 \\
Density & $\lambda_{\rho}$ & 1 \\
Strain & $\lambda_{\mathrm{L}}{ }^{0.5}$ & 10 \\
Time & $\lambda_{\mathrm{L}}{ }^{0.75}$ & 31.6 \\
Stress & $\lambda_{\mathrm{L}} \lambda_{\rho}$ & 100 \\
Displacement & $\lambda_{\mathrm{L}}{ }^{1.5}$ & 1000 \\
Velocity & $\lambda_{\mathrm{L}^{0.75}}$ & 31.6 \\
Acceleration & 1 & 1 \\
Frequency & $\lambda_{\mathrm{L}}{ }^{-0.75}$ & 0.03 \\
Pore fluid viscosity & $\lambda_{\mathrm{L}^{-0.75}} \lambda_{\rho}$ & 0.03 \\
Shear wave velocity & $\lambda_{\mathrm{L}^{0.25}}$ & 3.16 \\
\hline
\end{tabular}

${ }^{*}$ Item in prototype divided by the same item in model

Table 2: Soil properties.

\begin{tabular}{lcccccc}
\hline \multicolumn{1}{c}{ Soil } & $\mathrm{G}_{\mathrm{s}}$ & $\mathrm{e}_{\min }$ & $\mathrm{e}_{\max }$ & $\mathrm{D}_{50}$ & $\mathrm{C}_{\mathrm{u}}{ }^{*}$ & $\mathrm{C}_{\mathrm{c}}{ }^{\dagger}$ \\
\hline Ottawa sand & 2.66 & 0.61 & 0.79 & 0.19 & 1.69 & 1.01 \\
Construction sand & 2.66 & 0.46 & 0.81 & 0.25 & 1.87 & 0.91 \\
\hline \multicolumn{7}{c}{ Coefficient of uniformity } \\
& ${ }^{+}$Coefficient of curvature
\end{tabular}

Table 3: Variations of cohesion with temperature for a silty sand (Tsytovich 1975).

\begin{tabular}{cccc}
\hline & $-0.4^{\circ} \mathrm{C} \leq \theta \leq-0.3^{\circ} \mathrm{C}$ & $-1.2^{\circ} \mathrm{C} \leq \theta \leq-1.0^{\circ} \mathrm{C}$ & $-4.2^{\circ} \mathrm{C} \leq \theta \leq-4.0^{\circ} \mathrm{C}$ \\
\hline$c_{\theta}(M P a)$ & 1.1 & 1.4 & 2.0 \\
\hline
\end{tabular}

Table 4: Uniaxial compression resistance of structurally undisturbed permafrost (silty sand) (Tsytovich 1975).

\begin{tabular}{ccc}
\hline Moisture content (\%) & Temperature $\left({ }^{\circ} \mathrm{C}\right)$ & Strength $(\mathrm{MPa})$ \\
\hline 19.8 & -1.3 & 10.3 \\
19.1 & -3.9 & 13.7 \\
19.8 & -12.0 & 17.1 \\
29.3 & -11.0 & 9.5 \\
\hline
\end{tabular}

Table 5: Configurations of soil models used in experimental program (all dimensions are in $\mathrm{cm}$ ).

\begin{tabular}{cccccc}
$\begin{array}{c}\text { Experiment } \\
\#\end{array}$ & $\begin{array}{c}\text { No. of } \\
\text { Blocks }\end{array}$ & $\begin{array}{c}\text { Total thickness } \\
\text { of soil } \\
(\mathrm{H})\end{array}$ & $\begin{array}{c}\text { Frozen block } \\
\text { thickness } \\
\left(\mathrm{H}_{\mathrm{f}}\right)\end{array}$ & $\begin{array}{c}\text { Frozen block } \\
\text { width } \\
\left(\mathrm{W}_{\mathrm{f}}\right)\end{array}$ & $\begin{array}{c}\text { Span length } \\
\left(\mathrm{W}_{\mathrm{u}}\right)\end{array}$ \\
\hline 1 & 0 & 40 & - & - & - \\
2 & 1 & 40 & 20 & 10 & - \\
3 & 2 & 40 & 10 & 10 & 50 \\
4 & 2 & 40 & 20 & 10 & 50 \\
5 & 2 & 40 & 38.75 & 10 & 50 \\
6 & 2 & 40 & 20 & 10 & 30 \\
7 & 2 & 40 & 10 & 10 & 30 \\
8 & 2 & 40 & 10 & 10 & $10-50 \dagger$ \\
\hline
\end{tabular}

